\title{
Institutional Review Board Approval as an Educational Tool
}

\author{
Richard Balon ${ }^{1}$ (D) - Anthony P. S. Guerrero ${ }^{2}$ John H. Coverdale ${ }^{3}$ - Adam M. Brenner ${ }^{4}$ - Alan K. Louie ${ }^{5}$. \\ Eugene V. Beresin ${ }^{6} \cdot$ Laura Weiss Roberts $^{5}$
}

Received: 15 January 2019 / Accepted: 18 January 2019/Published online: 31 January 2019

(C) Academic Psychiatry 2019

Conducting a research study is usually a complex enterprise requiring a number of steps, starting with the initial idea and formulation of a research question, followed by the literature review, writing up the protocol, and then conducting the study. One of these steps is obtaining approval from the institutional review board (IRB) or a formal statement that the research is exempt from IRB oversight. (Other names used for institutional review bodies are, for example, ethics committee, independent ethic committee, ethical review board, research ethics board, human subjects committee, or human studies committee.)

The IRB review has two critical goals: ensuring the adequate protection of volunteers in human studies ("do no harm") and enabling a scientific process by which new knowledge may be rigorously acquired, bringing potential benefit to the public and society broadly ("help others"). A third goal, added by some, is protection of the institution from litigation.

In our role as editors for Academic Psychiatry, we often field questions regarding the necessity of IRB approval for papers based on projects involving educational data or educational cases. We have learned that many of our colleagues are unaware of the need for prospective IRB approval or formal exemption for education-related studies, and we have seen that many more of our colleagues view the process of seeking approval from an IRB as burdensome, time-consuming, or intimidating. In this editorial, we wish to reaffirm the

Richard Balon

rbalon@wayne.edu

Wayne State University, Detroit, MI, USA

2 University of Hawai'i John A. Burns School of Medicine, Honolulu, HI, USA

3 Baylor College of Medicine, Houston, TX, USA

4 University of Texas Southwestern Medical Center, Dallas, TX, USA

5 Stanford University, Stanford, CA, USA

6 Harvard Medical School, Boston, MA, USA importance of prospective IRB review, leading to approval or formal exemption for projects conducted by our authors, before submitting manuscripts to Academic Psychiatry. Toward this objective, we further wish to emphasize a less well-appreciated function of the IRB approval process, namely, to serve as a pedagogical resource for investigators, especially for those who are new to the conduct of human studies and research in general. We will discuss the critical functions of IRBs and how the IRB application processes can promote learning, particularly regarding clarity of writing, formulating research questions, and the ethical imperatives of research.

\section{The Role of IRBs}

The main role of the IRB is oversight of research to make sure that it is ethical and that human research subjects are appropriately protected from physical and psychological harm. The IRB also addresses safeguards and related issues, such as the process of informed consent, protection of confidentiality, management of conflicts of interest, evaluation and tracking of reports of adverse outcomes, and continuous surveillance of compliance with research regulations. A similar body called the Institutional Animal Care and Use Committee oversees funded animal research.

In the USA, IRBs are governed by the Code of Federal Regulations, Title 45, Public Welfare, Subtitle A, Department of Health and Human Services, Subchapter A, General Administration, Part 46, Protection of Human Subjects, known as the Common Rule [1]. (The Common Rule was recently updated, and the updated version was consulted for this editorial.) The regulations outlined in the Common Rule originated in the National Research Act of 1974 enacted by the U.S. Congress and the Belmont Report [2], written by the National Commission for the Protection of Human Subjects of Biomedical and Behavioral Research. The Belmont Report outlined the ethical principles guiding research including human subjects: beneficence, justice, and respect for persons. IRBs in the USA are regulated by the 
Office for Human Research Protections within the U.S. Department of Health and Human Services (HHS) and the Food and Drug Administration (FDA). The FDA has its own code of federal regulations [3], which is nearly identical to the HHS Common Rule.

The research ethics committees in countries other than the USA are usually guided by the Declaration of Helsinki [4] developed by the World Medical Association as a statement of ethical principles for medical research involving human subjects, including research on identifiable human material and data.

\section{Types of IRB Reviews and Approvals}

Some categories of research are exempt from IRB oversight. These categories include most educational research, research analyzing existing data and other materials that are already publicly available, and research evaluating the effectiveness of public benefits or service programs, efforts evaluating quality improvement of systems or programs, or evaluation of food taste and/or acceptance. Research that qualifies for exemption does not involve clinical interventions with human subjects, which has risks. In some educational research, adverse outcomes may result from exposing subjects to educational programs, such as situations that may trigger adverse emotional responses, or using a methodology that may not ensure anonymity. It is important to note that while some areas of research are exempt from IRB oversight, it is the IRB - and not the investigator - who determines the appropriateness of a formal exemption. The revised Common Rule does not necessarily direct how exemptions are determined by the institutions. The Office for Human Research Protections, however, continues to state that investigators are not authorized to make a determination that their own human subjects research is exempt because of the potential conflict of interest. The exempt category does not mean that the research is exempt from IRB approval; it means that the research is exempt from further review. According to the new version of the Common Rule, some of the exempt research actually may be required to undergo limited IRB review (e.g., to ensure adequate confidentiality and privacy safeguards). Thus, it is essential and critical, always, to obtain a determination from the relevant IRB regarding exemption of one's research. Even quality improvement studies may require IRB evaluation if the intention, eventually, is to publish the findings of these evaluative processes (i.e., "contribute to generalizable knowledge" [1]).

Studies that are not deemed exempt could be reviewed through two types of processes: at a full board or convened meeting by the IRB committee (a majority of members must be present) or by an expedited review process that is usually carried out by the IRB chair (or the chair's designee). Research considered to be of minimal risk typically is appropriate for expedited review. Research protocols can be either approved or disapproved, and the principal investigator can be asked for either minor revision (possibly reviewed further only by the IRB chair or designee) or major revision (reviewed further by the entire IRB committee).

It is important to realize that the consideration of protection of human subjects does not include only the "simple" protection from direct physical or psychological harm but also consideration of the merit of the planned research study. Volunteers in human studies (referred to as "human subjects" in federal regulations [1]), even those in educational research proposed in exempt status, should not be exposed to the risks of research without scientific merit. Thus, it is important to determine whether the project meets the definition of research and involves human subjects. Research is defined as "a systematic investigation, including research development, testing, and evaluation, designed to develop or contribute to generalizable knowledge" [1]. It is further defined as involving "human subjects" if it involves obtaining "information or biospecimens through intervention or interaction with the individual" or obtaining, using, studying, analyzing, or generating "identifiable private information or identifiable biospecimens" [1].

Many activities in which academic psychiatrists engage would not be viewed, formally, as "human subjects research." Literature reviews, program descriptions, expressions of opinions or perspectives, and other creative writings are clearly not human research or experimentation. Nevertheless, academic psychiatrists may also collect information or data - even identifiable, private information, for example, on student performance and feedback on faculty and curricula - in their usual activities (e.g., as required for program accreditation or quality improvement). Faculty members may collect information regarding students' reactions to educational curricula that may be highly sensitive, such as educational material involving sexual identity, cultural competence, or ethical issues in clinical practice. These activities are not necessarily "research" if there was no systematic investigation or intent to contribute to generalizable knowledge. However, there are times when faculty can see that information collected for one purpose may be helpful to others, and this moment is when IRB approval or IRB formal exemption should be obtained.

Use of information previously collected for another purpose, for example, data previously collected from people as an institution-focused quality improvement activity, that can be disseminated through scholarly presentations or publications now, importantly, qualifies for designation as human research, and consultation with the IRB is needed before publication or other forms of dissemination to broader audiences. At this point, regulations require engagement of the IRB, resulting in either a formal designation for exemption or formal approval of a research protocol. Under most circumstances, approval that was not obtained prospectively cannot 
be obtained retrospectively. (Some IRBs may differ in their approach to retroactive use of some data.)

The situation regarding the requirement of IRB approval may not always be unequivocal. Therefore, we believe that every protocol involving studying human subjects, whether the investigator considers it research or not, should be submitted to the IRB to determine its status and possibility of exemption from further review. Many journals, including Academic Psychiatry, require documentation regarding whether IRB review was sought, the name of the IRB, and the outcome (i.e., exempted from further review or approved). Reviewers of the research proposal need to see a full, well-written protocol that considers all aspects of protection of human subjects, including confidentiality, privacy, the possibility of obvious or subtle coercion, and other aspects to determine the category of IRB review and approval. Thus, each research protocol has to be carefully written, not only demonstrating adequate protection of human subjects but also explaining the merits of the study.

\section{Protocol Writing}

Protocol writing could serve as an educational tool in a number of areas, including clarification of the research question, critical review of the literature, clarity of writing, statistics, and adherence to ethical principles of human research. The following sections describe how the IRB application processes can promote learning.

\section{Clarification of the Research Question}

As mentioned earlier, human subjects should not be exposed to research without scientific merit. Thus, the protocol submitted to the IRB should be clear about the reason and focus of the research and its merit. It is also important to consider whether the design of the study will actually help to answer the research question. Determination of scientific merit is usually left to the department chair (or the chair's designee) or a special departmental committee, and the IRB requires that the chair, designee, or committee signs off on the research question as part of the submission. Even though the IRB's role is primarily the protection of human subjects, IRB reviewers may consider whether the research question is clear and whether the research makes sense from a broader ethical perspective. Consideration of the research question includes its importance, which may be judged in terms of whether and for whom one's research is beneficial.

\section{Critical Review of the Literature}

A critical review of the literature will help with clarifying the research question and justifying the study. Has the research question already been explored by someone? Was it probed enough? If it was probed, would replication be useful, beneficial? If the research question has not been probed, is there any literature suggesting that it may be useful to pursue it?

\section{Clarity of Writing}

The research protocol submitted to the IRB should be clearly written. Further, the rationale, protocol, and safeguards of the study should be conveyed in language that is understandable to someone who is outside of the researcher's area of expertise (e.g., committee members who are not psychiatrists) and to a layperson (IRBs are mandated to have lay members). A clearly written text of the IRB protocol submission may later help with writing up the study for publication.

Clarity of the informed consent form deserves special attention in the research protocol. Research volunteers must be able to read and understand the informed consent form and must be able to understand the benefits and risks to their participation and to appreciate that they can decline to participate without penalty. Both the Office for Human Research Protections and the FDA $[1,3]$ require the consent form to be "in language understandable to the subject or the legally authorized representative" [1]. The educational level of volunteers may limit their understanding of the informed consent. Thus, some IRBs require informed consent forms to be written at a certain educational level (e.g., a 5th or 6th grade reading level). It is important to realize, however, that in addition to difficult language, another limiting factor is the complexity of the science [5]. For some kinds of research, a consent form is not required, in which case a waiver of consent, granted by the IRB, will be necessary.

\section{Statistics}

IRBs may ask about statistics and may, in larger studies with complex outcomes, require a power analysis (i.e., a method helping to establish a sample size allowing a reliable statistical analysis and to determine whether one's statistical analysis will be able to detect the expected difference). For some researchers, this feedback from an IRB might require including a statistical consultant as a team member to ensure that appropriate scientific rigor is applied when analyzing the findings of the project. Considering the statistics that will be used goes back to the clarification of the research question and the ability of the research proposal to answer it. In particular, statistical considerations allow an assessment of whether the research design is sufficiently powered to detect a difference between groups when one truly exists.

\section{Adherence to Ethical Principles of Human Research}

Beneficence, justice, and respect for persons are the three main principles of human investigation, as set forth by the 
Belmont Report [2]. These principles characterize the intentions and practices of studies, and they translate concretely into safeguards that help protect study volunteers. More comprehensive discussions of the principles and safeguards are provided elsewhere $[6,7]$. In the following sections, we touch upon a few examples of safeguards as basic knowledge: definition and protection of human subjects, informed consent, and confidentiality.

\section{Definition and Protection of Human Subjects}

It is important to define who the subjects of the research proposal are and how they should be protected in light of the nature of the research. The protocol submission needs to specify the age and gender of the research subjects, inclusion and exclusion criteria, and whether members of vulnerable populations are a focus of the study. Representation of minorityidentifying individuals, especially in larger studies, also should be considered in relation to the generalizability of results and to ensure that a disproportionate burden is not placed on any particular population in seeking to answer a scientific question of importance to broader populations. A researcher should be able to state clearly whether and why the study population is appropriate, that is, whether the selection criteria adhere to the ethical principles of research and are suited to answering the scientific question that underlies the proposed work. The possibility of coercion also needs to be closely examined. Subtle coercion may occur in educational research; consider, for example, the pressure from program directors who repeatedly ask residents to participate in their study.

\section{Informed Consent}

Informed consent is a process that may or may not include a formal review and the signing of an informed consent form by the research subject. As Whitney states, "Consent enables subjects to enroll in research on their own terms and makes possible research that will benefit us all. Consent does not ... funnel more subjects into research; it helps each person make the choice that is right for him or her" [8, p. 3]. The IRB may waive a formal consent process in very rare situations that may not apply to educational research. The IRB may waive the use of informed consent forms, especially in cases involving no more than minimal risks or when the rights and welfare of the research subjects are not adversely affected (but again, this is up to the IRB's discretion). An informed consent form may be replaced by an information page outlining the study in simple terms to volunteers being recruited, a frequent method in Internet surveys, for example. The information page must include a statement that participation is voluntary and that answering the survey implies informed consent (e.g., a simple sentence may convey that answering the survey implies informed consent).

Preparing a protocol for IRB review helps the researcher decide about the process of informed consent and whether to use an informed consent form or an information page. It also helps when considering the risks and benefits of the study, because the IRB proposal requires spelling them out in the protocol and in the informed consent form or information page. We have already mentioned the importance of providing clarity and readability in the informed consent form. The risks and benefits have to be outlined in clear, understandable language, and highly technical terms should be avoided.

\section{Confidentiality}

Confidentiality is part of the protection of research volunteers. While preparing a protocol for IRB review, the researcher needs to consider how the data will be protected, stored, and coded. This aspect of research may seem to be a moot point for anonymous surveys, but confidentiality is not irrelevant in the case of small cohorts of residents or medical students, for example, and those steps need to be carefully spelled out when describing how the confidentiality of collected information will be protected.

\section{Writing Protocols for IRB Approval as an Educational Tool}

IRB review processes facilitate optimal and ethical research conduct - and they also educate investigators. In our role as editors of Academic Psychiatry, we often encounter misunderstanding of institutional review requirements as authors seek to submit their work for publication. Rather than viewing such duties as additional burden, we hope to persuade our authors and readers that the process of preparing documentation for an IRB and seeking approval for a proposed project can be an excellent educational experience. Table 1 presents additional recommended reading. Working in partnership with members of an IRB in seeking approval for a project may deepen one's understanding of a topic and highlight the need for additional knowledge and of different research designs and methods. All the elements of a scientific study - the elucidation of the research question, critical review of the literature, clarity of writing, statistics, and adherence to ethical principles, including the definition and protection of human subjects, informed consent, and confidentiality - are valuable. These features of a protocol may not be especially complicated in the case of educational research, and considering each of these elements is an excellent learning exercise, allowing for greater clarity of thought and of scientific and safeguard procedures. 
Table 1 Recommended reading

Arford PH. Human research protections: working with the IRB. Scotts Valley, CA: CreateSpace Independent Publishing Platform; 2015.

Mazur DJ. Evaluating the science and ethics of research on humans: a guide for IRB members. Baltimore, MD: The Johns Hopkins University Press; 2007.

Roberts LW. A clinical guide to psychiatric ethics. Arlington, VA: American Psychiatric Association Publishing; 2016.

Roberts LW, Geppert CMA, Coverdale J, Louie A, Edenharder K. Ethical and regulatory considerations in educational research. Acad Psychiatry. 2005;29:1-5.

Whitney SN: Balanced ethics review. A guide for Institutional Review Board members. New York: Springer; 2016.

\section{Compliance with Ethical Standards}

Disclosure On behalf of all authors, the corresponding author states that there is no conflict of interest.

Publisher's Note Springer Nature remains neutral with regard to jurisdictional claims in published maps and institutional affiliations.

\section{References}

1. United States Federal Government. Code of Federal Regulations, Title 45, Public Welfare, Subtitle A, Department of Health and Human Services, Subchapter A, General Administration, Part 46, Protection of Human Subjects. July 19, 2018. Available at https://www.ecfr.gov/cgi-bin/
retrieveECFR gp $=\& \mathrm{SID}=83 \mathrm{~cd} 09 \mathrm{e} 1 \mathrm{c} 0 \mathrm{f} 5 \mathrm{c} 6937 \mathrm{~cd} 9 \mathrm{~d} 7513160 \mathrm{fc} 3 \mathrm{f} \& \mathrm{pitd}=$ 20180719\&n=pt45.1.46\&r=PART\&ty=HTML. Accessed 15 Jan 2019.

2. The National Commission for the Protection of Human Subjects of Biomedical and Behavioral Research: The Belmont Report. Ethical Principles and Guidelines for the Protection of Human Subjects of Research. Department of Health, Education and Welfare Publication No. (OS) 78-0012; (30 September 1978); United States Government Printing Office, Washington, D.C.

3. United States Federal Government. Code of Federal Regulations, Title 21, Food and Drugs, Chapter I, Food and Drug Administration, Department of Health and Human Services, Subchapter AGeneral. December 20, 2018. Available at https://www.ecfr.gov/cgibin/text-idx?SID=1397a97a9e6c26b4e58857b25fcddcc $2 \&$ mc $=$ true\&tpl=/ecfrbrowse/Title21/21 cfrv1_02.tpl\#0. Accessed 15 Jan 2019.

4. World Medical Association. WMA Declaration of Helsinki - Ethical Principles for Medical Research Involving Human Subjects. July 9, 2018. Available at https://www.wma.net/policies-post/wmadeclaration-of-helsinki-ethical-principles-for-medical-researchinvolving-human-subjects/. Accessed 15 Jan 2019.

5. Whitney SN. Consent in biomedical research. In: Whitney SN, editor. Balanced ethics review: a guide for institutional review board members. New York: Springer; 2016. p. 47-56.

6. Roberts LW. Psychiatric research. In: Roberts LW, editor. A clinical guide to psychiatric ethics. Arlington: American Psychiatric Association Publishing; 2016. p. 237-66.

7. Roberts LW. Ethical dimensions of psychiatric research: a constructive, criterion-based approach to protocol preparation. The Research Protocol Ethics Assessment Tool (RePEAT). Biol Psychiatry. 1999;46:1106-19.

8. Whitney SN. Introduction. In: Whitney SN. Balanced ethics review: a guide for institutional review board members. New York: Springer; 2016. pp. 1-10. 